Original Research

\title{
Mothers' Strategies for Promoting Children's Healthy Eating, Physical Activity, and Screen-Related Behaviors
}

\author{
Jacy C. Downey ${ }^{1,}{ }^{*}$, Clinton G. Gudmunson ${ }^{2}$
}

1. Health Services Leadership, Simpson College, Indianola, lowa, USA; E-Mail: jacy.downey@simpson.edu

2. Human Development \& Family Studies, lowa State University, Ames, lowa, USA; E-Mail: cgudmuns@iastate.edu

* Correspondence: Jacy C. Downey; E-Mail: jacy.downey@simpson.edu

Academic Editor: Gerhard Litscher

Special Issue: Supporting Children with Integrative Techniques During and After Public Health Crises

OBM Integrative and Complementary Medicine

2022, volume 7 , issue 1

doi:10.21926/obm.icm.2201006
Received: October 19, 2021

Accepted: January 28, 2022

Published: January 29, 2022

\begin{abstract}
Parents model and teach early health practices that persist into adulthood by establishing a family culture through which children learn related family beliefs, values, and expectations. This phenomenological study examined the ways in which a purposefully selected group of mothers (selected due to their high scores on the Family Nutrition and Physical Activity screening tool) shaped family culture related to physical activity, addressed screen-time behaviors, and established positive eating related routines. Findings related to mothers' knowledge and belief systems about parenting within this domain pointed to the impact of family health history and mothers' own upbringing; a finding that reinforced the powerful nature of early habit formation. Mothers prioritized this parenting domain and were intentional in their efforts as they maneuvered threats from the obesigenic environment and worked to support their children's developing autonomy over related behaviors. This study presents a strengths perspective and imparts a new narrative which serves to complement existing obesity research in representative and at-risk populations.
\end{abstract}

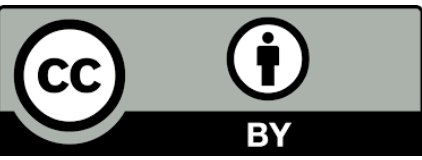

(C) 2022 by the author. This is an open access article distributed under the conditions of the Creative Commons by Attribution License, which permits unrestricted use, distribution, and reproduction in any medium or format, provided the original work is correctly cited. 


\section{Keywords}

Autonomy; beliefs; eating behaviors; knowledge; parenting; physical activity behaviors; screen-related behaviors

\section{Introduction}

Leading researchers in the area of parenting ecology and obesity have called for further study on the relationship between parenting practices and children's health behaviors. Much attention has focused on studying the obese population, however less research has been dedicated to understanding the nuances of behaviors of individuals who "resist environmental and lifestyle factors that promote passive and gradual weight gain" ([1], p. 1092). This phenomenological study makes meaning of the reflections, intentions, and strategies of a specific group of parents that are intentional in their efforts to positively socialize their children's eating, physical activity, and screenrelated behaviors. By utilizing purposeful sampling methods, this study presents an opportunity to provide a specific perspective and obtain a new narrative by giving voice to this overlooked population. Specifically, the purpose of this study is to provide detailed contextual description of the knowledge and belief systems of a select group of mothers as they intentionally shape their children's eating, physical activity, and screen-related behaviors amidst the threats that prevail in an obesigenic environment defined as "the sum of influences that the surroundings, opportunities, or conditions of life have on promoting obesity in individuals or populations" ([2], p. 564).

\subsection{Literature Review}

According to the most recent report by the National Health and Nutrition Examination Surveys (NHANES), 40\% of adults (BMI $\geq 30$ ) [3], and $19.3 \%$ of children ages 2 to 19 years-old (>95 $5^{\text {th }}$ percentile; [4]) are considered obese as compared to the early 1970 s when $4-6 \%$ of youth from 2 to 19 years-old were considered obese [5]. Current literature reports that overweight children become overweight adults $[6,7]$ and having obese parents is a powerful predictor of childhood obesity [8, 9]; a testament to the potent combination of shared genes, habits, and environment. As a result of threats from the current environment, obesity trends suggest that parents that do not intentionally socialize their children to have positive eating, physical activity, and screen-related behaviors may run a higher risk that their children will become overweight or obese [10]. Opportunities to obtain food and drink are plentiful, widely accessible, and convenient in non-third world countries [11]. This environment has encouraged higher caloric consumption, specifically through meals more commonly being consumed outside the home [12]. Children's diets-- characterized by highly tempting, low-nutrient, calorie dense foodstuffs-- do not align with the diverse and balanced meals needed to promote healthy growth and development. Additionally, the majority of American adolescents and adults are not sufficiently active [13], and Fakhouri and colleagues [14] found that fewer than 4 in 10 school-age children met both physical activity and screen-time recommendations. A systematic review of reviews found that high levels of screen time was associated with adiposity and unhealthy diet in children [15], and a growing body of evidence 
suggests that childhood sedentary behaviors and physical activity track into adulthood $[16,17]$. Without intervention, our children and their futures are in jeopardy.

\subsection{Health Socialization of Eating, Physical Activity, and Screen-Related Behaviors as a Parenting Domain}

Parents socialize their young by establishing a basis through which children (1) understand family beliefs, values, and expectations, (2) identify roles and responsibilities, and (3) develop suitable conduct [18]. Study of early socialization has found that varying contexts inspire dynamic parenting practices. Additionally, parenting practices that are domain-appropriate are more likely to be effective such that rules and expectations are designed to address specific behaviors (i.e., schoolwork, chores, etc.) rather than to be used as overarching statements [19]. Parental knowledge and beliefs about the parenting domain of obesity-related behaviors can be conceptualized as the attention and importance parents ascribe to healthy eating and activity, as well as specific knowledge about appropriate diet $[10,20]$ and activity. Likewise, parental beliefs are represented in their attitude towards the responsibility of embedding healthy habits in children, their consideration of children's competencies, and their perceptions of the risks that threaten goals for their children ([21], p. 300).

Parents are powerful socialization agents for shaping children's early eating behaviors, serving as role models [22], as well as managers and encouragers of their children's diet [23]. However, parental impact on children's eating practices is also influenced by contextual and environmental factors.

Parents are also influential in supporting their children's activity patterns through funding and providing access to opportunities that encourage physical activity [10,24], as well as maintaining an expectation of activity that enables children to perceive themselves as competent $[25,26]$. Parents also influence child physical activity by watching events, co-participating in activity, and reinforcing children's participation [27]. Parental support is especially important when children are not naturally inclined to be athletic or competitive as a means to help them build skills and confidence or find alternate ways to be active [28]. A conclusion drawn from 18 published reviews on studies of environmental correlates of weight-related behaviors in youth suggested that, unlike parental influence on eating practices, youth physical activity levels are not highly influenced by modeling, parental support, or home opportunities for physical activity. This finding is likely explained by the nature in which physical activity occurs (outside of the home) such that the influence of the home environment is reduced [29]. Parental socialization related to eating, however, may be more embedded into the lifestyles of families as food-related activities such as planning for, shopping, preparing, and consuming of meals are necessarily repetitive and time-consuming.

Although there is growing public health concern over obesity risks related to screen-related behavior in youth, parents reported greater concern about the content of the media their children are consuming rather than the amount, possibly explaining why parental concern has not translated into decreased viewing time in children [30]. Parents of high screen-time users, children that accumulate greater than two hours per day, reported fewer screen-related rules and a less negative attitude about screen use [31]. Households in which parents employ authoritative parenting practices, such that obedience is not simply demanded but rather boundaries are set, and children are reasoned with in a developmentally appropriate manner, promoted responsible decision- 
making around desired behaviors [32] such as children's screen use. In conclusion, in that overweight tracks from childhood into adulthood, early formation of eating, physical activity, and screen-related behaviors emerges as an area of focus to curb obesity trends. However, parents, as socializing agents, face many barriers that impact their ability to successfully guide children's eating, physical activity, and screen-related behaviors [10, 24]. Selection of a strength-based group of parents provides an opportunity for best practices to be uncovered and allows for the introduction of a new success narrative.

\subsection{Conceptual Framework: The Family Ecological Model}

The contribution of family ecology to children's eating, physical activity, and screen-related behaviors can be understood within the broader context of early socialization. The Family Ecological Model (FEM), based on the ecological systems theory and developed by Davison and Campbell [33] to understand the context in which parenting occurs, considers both immediate and broad influences impacting the ecology of parenting. The four immediate aspects of parenting that relate to obesity-related behaviors-- knowledge/beliefs, modeling, accessibility, and shaping-- illustrate how specific parenting processes influence children's health behaviors. These aspects are, in turn, influenced by broader contextual characteristics of the child, family, community, organizations, policies, and media [21]. As such, interviewed participants in this study were asked to describe (1) their beliefs related to children's obesity-related behaviors, (2) life events and external factors related to family of origin, and (3) education and work-related experiences that have shaped how they have become deliberate in this parenting domain.

\section{Methods}

The aim of a phenomenological study is to uncover the perceptions, perspectives, and understandings of a particular situation or phenomenon [34]. Examination of multiple perspectives of the phenomenon allows a researcher to understand an experience from the vantage of the beholder. As such, one-on-one interviews are the principal means of collecting data for phenomenological studies as they foster extensive interaction between researcher and participant and elicit "in-depth, context-rich personal accounts, perceptions, and perspectives" from individuals sharing a common experience [35]. To commence the data collection in this study, IRB approval and informed consent were obtained, and interviews were scheduled to be held in a private conference room located on the town square. An interview protocol inspired by the FEM guided interaction between researcher and participants. Consistent with phenomenological study, the FEM informed and supported the development of interview questions and contributed to final stages of analysis but did not constrain the development of new information. Initial interview questions were guided by a small set of "grand tour" questions [36] focused on parenting processes related to eating, physical activity, and screen-related behaviors. The idea was to get participants talking openly to expose rich details of each of the four immediate parenting practices used to socialize children's obesity-related behaviors. Then, more specific questions and probes, some of which inspired by broad contextual influences, were used to gain further understanding of the participants' experiences [35]. The interviews were audio-recorded, transcribed, and analyzed. Transcripts were checked against recordings in situations when data was unclear or ambiguous. An audit trail was 
recorded throughout all stages to capture the process of conceptualization, sampling, data collection, analysis, findings, and dissemination.

\subsection{Sampling}

Insight from a specific subset of parents was needed to understand the phenomenon of interest [35]; therefore, the researcher utilized purposive sampling techniques to target individuals particularly knowledgeable about the phenomenon under investigation. Although purposive sampling precludes generalizability, it is a preferred method to reach participants exhibiting specific characteristics that are willing to talk about their unique positions [35]. Approval was given by a district superintendent to forward the screening tool to parents in each elementary school. The correspondence letter was administered to parents of elementary students (kindergarten- $5^{\text {th }}$ grade) attending four schools (student enrollments were 419, 377, 429, and 458) in a small Midwestern city (population 15,000). This letter contained a key informant survey that identified parents meeting study criteria as well as (1) the behaviorally anchored rating (BAR) scale version of the Family Nutrition and Physical Activity (FNPA) screening tool [37], (2) demographic questions (i.e., marital status, educational level, employment, race, number and ages of children, household income), and ( 3 ) a request for contact information.

The FNPA assesses 10 risk factors associated with overweight/obesity in children, loads on a single factor, and has been shown to have good internal reliability (alpha $=0.72$; [37]). Parents were asked to select the response (on a 3-point scale) that most closely resembled patterns in their family. Examples of possible responses to the question on 'Screen Time' include "My child watches television or plays on the computer (or with video games) for more than 4 hours each day," "My child watches little television but plays on the computer or with video games for 2-4 hours each day," "My child doesn't play on the computer (or with video games) but watches television for 2-4 hours each day," "My child watches television or plays on the computer (or with video games) less than 2 hours each day." Total scores on the FNPA could range from 10-30, with higher scores indicative of healthier family environment and behaviors.

Parents had to have at least one child in elementary school to be administered the survey as the FNPA [37] has been shown to identify family behaviors and environments that may predispose children of elementary age to overweight. Additional children in the family may be any age or gender as long as one child in elementary school resided in the home. Targeted participants could be mothers, fathers, or other primary caregivers; however, of the 346 parents that completed the survey, those with top scores (score $>29 / 30$ ) in this study were mothers. Parents could elect to complete the survey on paper or electronically. Following is a breakdown of FNPA total scores and, in parentheses, the numbers of individuals with those scores: $\leq 20$ (19), 21 (14), 22 (28), 23 (22), 24 (43), 25 (65), 26 (48), 27 (55), 28 (31), and 29 (21). Researchers analyzed the distribution of FNPA total scores to ensure that the purposeful group of mothers selected for interviews were top-scorers and not simply outliers.

\subsection{Participants}

The sample for this study was selected based on high scores on the FNPA; a high score signified healthier home environment and parenting practices within this domain. A small percentage $(n=$ $21,6.1 \%)$ of parents qualified for the study based on recording the top FNPA score $(\geq 29 / 30)$. Of the 
21 qualified participants, 12 mothers responded to invitations and agreed to participate in two-hour in-depth interviews. In proceeding towards the final interviews, we noticed mostly repeating themes and fewer new ideas emerging in the content area. By the final interview, we concluded that we had reached a level of sufficient saturation [38] so as not to require further recruitment for the study. For their involvement, mothers participating in interviews were given a $\$ 15$ gift card to a local grocery store. Mothers' real names were replaced with pseudonyms.

The interviewed group of mothers was not especially diverse in regard to race/ethnicity, education, and socioeconomic status. All of the interviewed mothers were Caucasian, and within the 26 to 41 age range. An average of 2.5 children (age range: 1 month to 15 years) lived in the home, and all of the mothers were currently married except one who was divorced. Mothers' highest achieved level of education was counted as follows: some college or vocational training (1), associate's degree (2), bachelor's degree (5), master's degree (1) and advanced degree (3). Four mothers were not employed outside the home. Of the employed mothers, two were self-employed, four employed by someone else, and one was working and going to school. Half of the mothers worked in a health-related field. The modal household income was in the range of $\$ 80,000$ to 109,999 .

\subsection{Analysis}

In the first step of analysis, by attending to psychological detail and focusing on understanding the meaning of the participants' experiences, the researcher underwent an intentional analysis aimed at uncovering the essence of the phenomenon [39]. The researcher prepared for analysis by identifying meaning units: strings of words and sentences within the transcripts that conveyed important concepts. Relevant phrases were tagged with pertinent descriptive information to provide contextual support to each excerpt and meaning units were organized to provide a situated description of parent health socialization. The researcher considered the details of individual parts (meaning units) and how the parts relate to the overall participant circumstances, all while considering how the participants' perceptions, emotions, and behaviors impacted her experience.

In the second step, the researcher reduced the data into themes as elements clustered around central ideas within each interview and across participants. Essential characteristics of the phenomenon were discerned from the superfluous [39], while the widely held and essential elements of the shared experiences were preserved, allowing the researcher to grasp the essence of the experience [40]. Thirdly, input was sought from all participants with five participants responding to a request to complete member checks. The researcher provided participants with a detailed narrative describing themes and subthemes uncovered from the interviews and asked for comment on the researcher's depiction of the reflections, intentions, and strategies related to parenting within the studied domain. Additionally, two colleagues with doctoral training in qualitative research participated in peer-debriefing exchanges on multiple occasions during analysis. This practice helped to ensure that participant experiences aligned with the researcher's depiction and enhanced the accuracy of the interpretation [35]. In the final stages, external and previously held frames of reference were incorporated to determine how the conceptual framework and relevant concepts informed the analysis ([39], p. 172). 


\section{Findings}

\subsection{Theme 1: Childhood and Family History Provide Motivation}

Participants were asked to describe past experiences and events that have shaped them into the type of parent that stresses the importance of positive eating, physical activity, and screen-related behaviors. Mothers ( $n=11$ ) emphasized their own upbringing as strong motivation to either parent similarly or to parent differently in response to their own childhood experiences. Most mothers noted that parental influences during development were highly influential in not only forming their own personal habits but also in inspiring them to focus on instilling positive habits in their own children. Jo, a mother of three, reflected on the impact of her own childhood, "My parents made it easier for me to make the right choices and so why not do it [for my children] now so then they can make the right choices easier in the future." Mothers agreed that having healthy habits had become natural over time, however some noted that they experienced a time of rebellion against their structured upbringing related to eating, but maturity eventually graced them with respect and appreciation of their parents' efforts and inspired them to keep some practices, establish new ones, and be rid of others in their own families. Many noted that, although they were raised with particularly strong models of positive eating habits, their parents did not always participate in regular physical activity.

Many mothers $(n=8)$ remarked of diabetic, heart-diseased, and obese family members making an impression on them whether it was the way the ill member(s) impacted their parents' formation of "house rules" or more directly on the participants themselves. References to struggle with weight, disease, or illness of close family members were commonly noted as motivation for their own parents' convictions, as described by Jo:

She [my mom] wanted to watch my dad's health because my grandma is diabetic and my dad is diabetic. My mom's always saying, 'you can't have this in the house'. Even from a young age we were really aware, I guess. My parents would make us aware, 'eating this will cause this and eating this will cause that'. My grandpa had a heart attack when I was pretty young, so I think that kind of woke up both of my parents a little bit more. That's when they were really big on healthy eating . . .well bigger, I guess.

The less dominant theme of a history with poor body image $(n=4)$ emerged as a motivating factor for mothers' emphasis on healthy habits. One mother, Leigh, recalls being labeled as "fat and bad at sports" as the beginning of a lifelong struggle with negative body image that she believes to have led her to give up on healthy habits and succumb to weight gain. She later adopted positive habits but continued to struggle to manage negative self-talk and her weight and was careful to orchestrate related conversations with her daughters, "They may not be [athletic], but I want them to know that they can use their bodies to do what they want to do and that other people can't tell them what is good or bad or otherwise." While the majority of mothers had a history of youth athletic participation, some of the mothers continued to regularly exercise as adults, for weight control as well as out of enjoyment. Mary, mother of two children, reported a sense of control over her issues with poor body image through eating healthily and participating in regular exercise. Many mothers noted periods of weight gain, poor body image and, ultimately, maturity as impetus for returning to or adopting positive behaviors. 


\subsection{Theme 2: Mothers Personally Value Positive Health Behaviors}

Mothers personally valued the short and long term benefits they believed to be associated with living a healthy lifestyle $(n=12)$. Mothers referenced a strong desire to live differently than family members and others diagnosed with lifestyle-related diseases, sharing multiple references to avoiding obesity, diabetes, cardiovascular disease, mobility restrictions, etc. as motivation for engaging in healthy behaviors. Dawn, mother of two boys, discussed how health concerns of family members provided motivation for supporting healthy habits in her children, "My mom has diabetes. Chris' [her husband] parent's both have diabetes . . . type II . . . because they're obese. So I do think that that plays a huge part in how we want to make sure that genetically they [their sons] are not more prone."

Mothers described themselves as personally valuing a healthy diet and, more commonly, regular physical fitness. Mothers' sentiments were characterized by a passionate interest in health behaviors and actively seeking related information, ranging from taking a recent college course to reading academic health articles to scouring blogs. Approximately half of the mothers $(n=5)$ either currently or previously were employed in a health-related field and some of their spouses $(n=3)$ are also employed in the health field, supporting the idea that mothers that are personally interested in health may find it easier or more natural to parent intentionally in this domain.

Mothers realized the physical, mental, and emotional benefits of engaging in healthy behaviors. Mothers described the way adopting regular exercise into their routine makes them feel, "It's almost like a drug... the endorphins you get make you feel good and you almost just need that every day." Steph talked how she became devoted to living a healthy lifestyle,

I think that when are you are healthy and you are fit you feel good and that, in and of itself, becomes kind of addictive. When you know what it's like to feel really good, why would you ever want to go back to feeling awful?

Steph later described the process of becoming healthy as a transition from lethargic to "euphoric" and announced it as an "aha moment" when she made the connection between her health-related habits and the way she felt physically, mentally, and emotionally. Other mothers ( $n$ $=11$ ) described exercise as stress relieving and empowering while many praised physical activity as a source of pride, self-esteem, and self-confidence.

\subsection{Theme 3: Focus on Health Intensified Upon Becoming a Parent}

Mothers ( $n=12$ ) explained that parents are charged with teaching their children what they know to be good and right, therefore, by knowing how important it is to have healthy behaviors, they were responsible for fostering such values in their children. Likened to other parental roles such as teaching their children right from wrong, mothers sensed the fleeting nature in which they were the primary influence in this realm of their children's lives. One especially resilient single mother of two boys, Railene, spoke of the need to build a solid expectation when her boys are young so they would be strong enough to make good decisions in the face of the temptations and outside influences that would increase with age. Another mother, Dawn, commented on the need for guidance on forming healthy lifestyles,

I don't think it's [incorporating regular physical activity into their lives] something that's just innate in us. I think that it's something that they have to learn. As toddlers they're moving all the time and it's not like they have to learn how to exercise, but as they get older they're more 
sedentary ... reading or being in front of a screen or hanging out with friends. I think that they need to learn how to incorporate that [physical activity] into their lifestyle.

Similarly, another mother of three young boys, Ann, agreed that children need to be taught about health habits as they were not naturally going to make good choices, "Otherwise [without parents taking this on as a responsibility], how are they going to know?"

All mothers $(n=12)$ identified the positive socialization of their children's health behaviors as a top priority, while Kristie described this as an area in which she "cannot fail." Mothers described the responsibility as urgent, commenting that, "When they're young they learn to eat for when they're older. It [positively socializing children's behaviors] should be a big priority, a lot bigger than what it is." One mother described how living their priorities meant absolute intentionality, such that all actions and words supported her and her husband's child-rearing goals. This same mother, Steph, credits her success to eliminating that which distracted her from her goals. Her family's busy life forced her to focus on only the highest of priorities, noting that, "I'm deliberate in everything, and it's so scheduled because we only have this little tiny window of time." Marie, a mother of two young children remarked on how she is continually striving to find ways to be deliberate in reaching her parenting goals in anticipation of fending off outside influences. When considering the upcoming baseball season which finds the family at the baseball fields multiple nights each week, one mother projects, "I'm going to have to start planning when they're in those activities. You have to be more clever with dinner . . use a crock pot more."

Mothers emphasized that they wanted healthy habits to be more than just another rule to follow. While some mothers believed their children were too young, many mothers $(n=9)$ believed their children had internalized the importance of positive health habits. Aspen, mother of three, described her goals, "[Understanding why is] very important because sooner or later they're leaving my house. I want it to be important to them, too, so that they continue on and ... they don't just do it now because I say they have to." Mothers, however, were careful to respect the specific stage of their children's development $(n=12)$ as they were aware of the age-related differences in which they were able to affect internalization. When children are younger, providing reasons for certain "house rules" are often unnecessary, as acting simply out of habit, being happy to please mom, or "because it's good for you" may be sufficient to achieve compliance. In regard to physical activity, Marie, a mother of younger children, remarked that often the "fun factor" is enough to entice younger children to be active but may not be enough inspiration for a lifetime,

Even if it's just us playing tag in the basement ... that was fun. We all laughed and Wilbur [the dog] chased us and [the kids] associate it with fun." [As they grow older] I hope they find an interest. It doesn't have to be an actual varsity sport or anything but something that keeps them active because I think [older] kids.... they're less likely to go just work out for fun.

Repetitively reinforcing the reasons for making healthy choices throughout development reinforced mother's values and supported internalization by aiding children as they made associations between house rules and desired behaviors. Aspen described the value of a lesson on nutrition she shared with her daughter while making dinner, "I think it helped connect the dots on why, 'we're not just being mean or grumpy or not letting you have macaroni and cheese because I want to make your life miserable'."

Marie described a typical interaction in regard to curbing screen-time, demonstrating how she manages pushback while nudging her young son toward internalization by redirecting his attention to matters important to him. 
'Mom you're so mean. Why don't you ever let me watch TV as long as I want to watch TV? When I get bigger I'm going to watch TV all day long'. That's what he always says and I tell him, 'it's not good for you to sit and watch TV. Your muscles want to be running and active.' [This tactic works] because he's so into that [being big and strong] right now.

As outside influences became more pervasive, mothers were careful to use age appropriate language and present reasons in ways that cater to children's interests. Julie, mother of three, reflects on previous conversations, "My kids are athletes so we talk about 'what do you need to eat to have enough energy to go do what you have to do'." In commenting on the growing influence of non-parental sources as children age, Ann discussed school food, "I think they [parents] have all the power, most of the power, I think we lose a little bit in the school system. I think it would help if schools ... if they really only offered healthy fresh-prepared meals ...I think it would be a lot easier to form those habits rather than to try to break them here [at school]."

\subsection{Theme 4: Acceptance of the Extra Effort}

While some exhibited positive habits prior to parenthood and did not have to undergo major lifestyle changes to "live" their priorities for the benefit of their children, all mothers noted that great energy, a strong commitment, sacrifice, and even sometimes feeling stigmatized was part of the process of embedding and supporting internalization of desired behaviors. Mothers $(n=12)$ spoke of time and effort invested in modeling appropriate behaviors, transporting children to activities to participate in physical activity, planning for healthy family meals around activities, and the constant juggling of the busy schedules of multiple children.

Based on the nature and sheer repetition of feeding younger children, mothers recounted that introducing and encouraging healthy eating practices was tedious. Mothers reported that less effort was required to engage children in physical activity as they were considered by many mothers to be naturally active. Ann, mother of three young boys, explained, "It's a battle to eat healthy sometimes, but it's not a battle to go out and play or do swimming lessons and things that are going to keep them active. They like it." Managing barriers and logistics became more of a challenge as children grew older and were more involved in structured physical activity and sports. Julie described how she guided her children as they packed lunches and snacks in preparation for an all-day swim meet in which food choices would be limited to the concession stand,

[I would say] think about what you're going to need to eat to keep your body going while you're swimming ... It's really hard at those events because it's boring for them when they're not swimming. They just want to sit and eat because what else do you do? You know you're sitting for eight hours ... so at least I know if we limit what they take, and it's all good choices, then if they eat it all in the first two hours of the day ... well, it isn't like they ate six bags of chips.

Mothers ( $n=12$ ) commonly remarked that early diligence and consistency were valuable in laying the groundwork for developing healthy habits. Marie, mother of three, remarked that remaining committed is difficult, "I think self-discipline is huge. All this stuff [healthy behaviors], in reality, sounds good and I'm passionate and believe it. But nothing helps unless you have self-discipline to integrate it. There are still some days where I don't want to."

Effort to set clear expectations and observance of house rules eventually resulted in diminished pushback from children, though mothers $(n=12)$ admitted to instances when limited time and 
energy challenged their convictions. Dawn, shrugged her shoulders and reminisced about earlier battles with her children, "It's harder to say no than it is to say yes."

Time is one of the things that make[s] it hard. Everybody's busy all the time, and there are days when it would be so much easier to go to the drive thru at McDonalds and that be their dinner than to go home after a long day and cook. I'm constantly having to make the choice, 'is this really what I want to do'? Some days tired wins out and you get something [away from home] for supper and that's just how it is. I think time is the biggest [barrier] because being healthy is a huge time commitment. It's conscious decisions every day. That's what it comes down to. You just are making a conscious decision every day to be healthy.

Mothers were frustrated that healthy foods were not often convenient. Multiple references were made to the many times it would have been easier to just "drive thru" than to plan, shop for, and prepare a healthy meal. Mothers stressed that parenting for healthy behaviors was complicated by our current environment, one in which temptation is inevitable. Frustratingly, Julie described a typical night at the ball fields, "Junk is everywhere. Like the concession stand, 'we're going to have fried cheese curds and donuts and French fries'. Who needs to sit at the little league field and eat that?" Though mothers concurred that they often had to forgo ease and expediency in meals, or give up free time for their own or their children's physical activity, these mothers accepted sacrifices as part of parenting. Marie provided a glimpse into her attitude about such sacrifices when she commented, "Those [making sacrifices] are all true factors and for anything we do."

Ann described how the strictness of screen-time monitoring was adjusted based on competing duties, "They'll watch their TV shows that they like probably a little more than I should be letting them. Sometimes it's hard to keep them entertained when you're tired or you have laundry to do or something like that." Mothers also described an internal struggle in regard to monitoring screentime. Screens presented a contradiction for many mothers, as the appropriate balance between allowing access to educational tools and limiting addictive and sedentary-promoting agents was continually evolving.

Beyond the sacrifices associated with positively socializing their children's eating, physical activity, and screen-related behaviors, some mothers described feeling a sense of stigma $(n=8)$. Not all mothers were bothered by criticism from others, however all mothers admitted that it was evident that their family "did things differently" than most. Steph described the harsh criticism she feels from others, "I think it's easy to blame ... to point fingers at somebody that has something that you want but you haven't figured out how to get." Another mother, Mary, described reactions from others, "I think people think it's pretentious. That's what I get from them. 'I'm not trying to be pretentious, just healthy'." Many mothers felt a sense of scrutiny when limiting children's intake of unhealthy items. Mothers disagreed with the premise of children's unrestricted ingestion of junk food and felt they were battling a cultural norm, as it is not socially acceptable to restrict children's consumption. While mothers felt that the current environment was not supportive of their efforts, the resolve of this select sample of mothers was captured when Steph proclaimed, "If I'm going to let this be an obstacle that's my fault. They [obstacles] are only as big as you make them."

\section{Discussion}

This qualitative analysis utilized purposive sampling to increase understanding of parental cognitions, behaviors, strategies, and responses to family and environmental dynamics used by a 
specific type of parent to socialize children's obesity-protective behaviors. This paper answers calls for research to better understand the context in which parents intentionally socialize children's obesity-protective behaviors while living within a non-supportive environment.

Aligning with the FEM [33], mothers' knowledge and beliefs about obesity-related behaviors were important determinants in shaping the ecology of parenting in this domain. Mothers believed that positively socializing their children's eating, physical activity, and screen-related behaviors required extensive time, energy, sacrifice, and commitment. However, by naming this area of socialization as a top priority and aligning their attention to this parenting domain, mothers justified the effort required to dedicate themselves to this responsibility. The insight provided by studying this population may inspire future research to consider how parental value systems may influence parenting strategies and practices across other domains.

Mothers reflected on their own upbringings as motivation for their commitment, reinforcing the powerful nature of early parent-child relations on behavior formation. Though behaviors established in their own childhoods were influential, mothers acknowledged the environment in which they are currently parenting as vastly different from their own, requiring regular attention and reevaluation of their focus and strategies. While firmly committed to establishing patterns in support of related goals for their children, mothers were also attentive to the dynamic needs and competencies of individual family members as well as the evolving influence of risk factors from outside the family unit (i.e. school, media, social norms; [21]) as suggested by the FEM. Mothers agreed that positively influencing their children's obesity-related behaviors was hindered by cultural and environmental factors (i.e. wide availability of problem foods and passive entertainment), however the mothers in this purposefully selected sample were adamant that what was expected and enforced within their home could stand up against outside influence. Additionally, mothers anticipated outside threats to increasingly become more widespread and complicated as children developed; therefore, mothers acted deliberately in preparation for them. This belief provided mothers timely motivation to build strong expectations in the early years when children are most impressionable to parental influence [18]). These findings highlight the need for contextual insight of elements outside the family environment that influence parental socialization in this domain, and specifically to understanding the competing nature of home and external factors on related child behaviors.

For these mothers, the creation of a specific family culture was vital to the process of guiding children's habits. Shaping family culture meant personally embodying valued behaviors. These findings support previous research on parental nutrition knowledge and modeling as determinants of child eating practices [10]. Although other work suggests that the impact of home environment on youth physical activity is not as substantial as with eating behaviors [29], mothers in this study strongly attributed their success in positively socializing their children's physical activity habits to parental modeling. Mothers spoke not only of providing a good example of desired behaviors but also of consistently nudging their children towards internalization of the short and long-term physical, mental, and emotional benefits of physical activity. Mothers' strategies included deliberate conversations as well as creating an "active" mentality such that family entertainment was regularly structured around physical activity rather than the more sedentary pursuits that characterize much of family entertainment.

The importance of the concept of internalization, a process through which one discovers value for an activity that was previously performed for other reasons, was an emergent finding in this 
study [41]. Motivation for a specific behavior may arise from regulation of the behavior, acting through external controls (parent pressure or use of rewards and punishment) which results in weak internalization or to a deeper form of internalization in which the child acts with a sense of volition such that the activity is autonomously controlled [41]. Mothers in this purposive sample described a desire to help their children internalize the value of positive behaviors and acted with this goal in mind. Previous research on parental support of autonomy suggests that parents support internalization best when embracing the perspectives of their children and acknowledging their feelings such that they use reasoning and allow choices over simply demanding obedience [42]. The influence of autonomy development on physical activity has been described [43], however further investigation on parental support of child autonomy across other behaviors associated with obesity may be warranted. Parental support of autonomy was not specifically outlined in the FEM but can be considered a dimension of parental knowledge and beliefs-at least in this sample of mothers. Future study might also consider how dynamics associated with child development and changing threats from the environment may impact how parents are able to support positive obesity-related behaviors becoming autonomously controlled by children. It is unclear if this finding is transferable to other populations, therefore future study might specifically address the degree to which other samples of parents support children becoming autonomous in obesity-preventive behaviors.

\section{Limitations}

This select group of mothers qualified for the study by posting high scores on the FNPA screening tool [37], shown to identify family behaviors and environments that may predispose children of elementary age to overweight. While the study design allowed mothers, fathers, and other primary caregivers to participate, $88.8 \%$ of those surveyed were mothers, and all participants meeting criteria to be interviewed were mothers. The voices of fathers were notably absent from this study. Literature suggests that mothers more commonly communicate with their children's school [44], and females are more likely to participate in survey research [45]. Therefore, as participants were reached through their children's schools, fathers were effectively removed from the pool of possible survey respondents, limiting transferability of findings solely to mothers.

Demographic factors may have also played a part in the inability to recruit a more diverse sample. Thus, findings were limited by the homogenous characteristics of this select group of parents in this sample and do not allow for the impact of commonly studied sociodemographic variables to be fully understood. By nature, phenomenological studies are not designed to be generalizable to greater populations; however, it is worth noting that while we did not intend to collect viewpoints from a non-diverse sample, interviewed participants were white, and generally well-educated and socioeconomically privileged women. Considering the wide prevalence of this epidemic, and, specifically, that white middle and upper income mothers are not protected from the threats of the obesity epidemic, we maintain that their experiences are important and their voices justified. Although low-income individuals are more commonly the target of obesity research as living in poverty is undoubtedly debilitating, many obese adults are not low income [6]. According to 2017 NHANES report, the most up-to-date publication of national obesity statistics, $39.0 \%$ of all obese adults reported incomes below $130 \%$ of poverty level, $40.8 \%$ had incomes between $130-350 \%$ of the poverty level, and $31.2 \%$ had incomes at or above $350 \%$ of poverty level [46]. 
While race, ethnicity, and socioeconomics play a part in the epidemic, the prevalence of obesity is growing at alarming rates regardless of these factors, making these mothers' strengths perspective a valuable viewpoint to share in the literature. Perhaps mothers that were able to ensure that the basic needs of their children were met were more able to dedicate time and energy to obesity-preventive practices. Future research should work to more clearly understand how privileged status may influence parental socialization of obesity-preventive behaviors as favorable resources and environments may contribute to the likelihood of practicing purposeful parenting in this domain. Additionally, studies designed to capture the voices of diverse families are warranted.

\section{Implications}

Hearing the voices of parents that are committed to fostering and maintaining practices that are known to be obesity-preventive provided a new narrative that lends limited yet unique insight into the complex health socialization domain. As best practices are commonly integrated into applied settings, efforts can be informed by a better understanding of the considerable influence that parents may have when positively socializing said behaviors is prioritized. Insight from these mothers could be incorporated into education directed to public health initiatives, pediatricians, primary care physicians, and nurses about the importance of stressing intentionality to parents of young children to inspire the important work of setting expectations and developing positive obesity-related behaviors. Ultimately, widespread education directed at the public, parents, and health professionals is needed to change the tone surrounding this important parenting domain.

When we draw from the findings of the present study and our understanding of the literature, it is clear that some parents have strong feelings about their role in the socialization of health behaviors in their children. This, however, required intentional effort and even great struggle at times. Although parents we interviewed could usually identify specific strategies that they had implemented in their own families to promote healthy child behavior, most often these tended to be idiosyncratic practices that would conceivably only work in very specific family settings. While it might not be warranted for educators to strip particular parenting strategies out of their context and promote them universally, practitioners might showcase strategy ideas as a means to seed creative thinking and to encourage parents to engage their children in the fight against obesity in ways that they can rally behind.

\section{Author Contributions}

The authors confirm contribution to the paper as follows: study conception and design: JD, CG; data collection: JD; analysis and interpretation of results: JD, CG; draft manuscript preparation: JD, CG. Both authors reviewed the results and approved the final version of the manuscript.

\section{Competing Interests}

The authors have declared that no competing interests exist.

\section{References}

1. Davison KK, Birch LL. Lean and weight stable: Behavioral predictors and psychological correlates. Obes Res. 2004; 12: 1085-1093. 
2. Swinburn B, Egger G, Raza F. Dissecting obesogenic environments: The development and application of a framework for identifying and prioritizing environmental interventions for obesity. Prev Med. 1999; 29: 563-570.

3. Hales C, Carroll M, Fryar C, Ogden C. Prevalence of obesity and severe obesity among adults: United States, 2017-2018. Hyattsville, MD: National Center for Health Statistics; 2020; No. 360.

4. Fryar CD, Carroll MD, Afful J. Prevalence of overweight, obesity, and severe obesity among children and adolescents aged 2-19 years: United States, 1963-1965 through 2017-2018 [Internet]. Hyattsville: National Center for Health Statistics; 2020. Available from: http://www.publicnow.com/view/57BFCB292A6D12A9A3EE633921C052DED8F0D94B.

5. Fryar CD, Carroll MD, Ogden CL. Prevalence of obesity among children and adolescents: United States, trends 1963-1965 through 2009-2010 [Internet]. Hyattsville: National Center for Health Statistics; 2012. Available from: http://www.nccpeds.com/ContinuityModulesFall/Fall\%20Continuity\%20Source\%20Materials/Obesity-2009-10\%20Trends.pdf.

6. Ogden CL, Lamb MM, Carroll MD, Flegal KM. Obesity and socioeconomic status in adults: United States, 2005-2008. Hyattsville, MD: National Center for Health Statistics; 2010; No. 50.

7. Ogden CL, Lamb MM, Carroll MD, Flegal KM. Obesity and socioeconomic status in children and adolescents: United States, 2005-2008. Hyattsville, MD: National Center for Health Statistics; 2010; No. 51.

8. Reilly JJ, Armstrong J, Dorosty AR, Emmett PM, Ness A, Rogers I, et al. Early life risk factors for obesity in childhood: Cohort study. BMJ. 2005; 330: 1357.

9. Zeller MH, Reiter-Purtill J, Modi AC, Gutzwiller J, Vannatta K, Davies WH. Controlled study of critical parent and family factors in the obesigenic environment. Obesity. 2007; 15: 126.

10. Savage JS, Fisher JO, Birch LL. Parental influence on eating behavior: Conception to adolescence. J Law Med Ethics. 2007; 35: 22-34.

11. Linde J, Jeffrey R. Evolving environmental factors in the obesity epidemic. In: Obesity epidemiology: From aetiology to public health. 2nd ed. Oxford: Oxford University Press; 2010. pp.119-135.

12. Zoumas-Morse C, Rock CL, Sobo EJ, Neuhouser ML. Children's patterns of macronutrient intake and associations with restaurant and home eating. J Am Diet Assoc. 2001; 101: 923-925.

13. Piercy KL, Troiano RP, Ballard RM, Carlson SA, Fulton JE, Galuska DA, et al. The physical activity guidelines for Americans. JAMA. 2018; 320: 2020-2028.

14. Fakhouri TH, Hughes JP, Brody DJ, Kit BK, Ogden CL. Physical activity and screen-time viewing among elementary school-aged children in the United States from 2009 to 2010. JAMA Pediatr. 2013; 167: 223-229.

15. Stiglic N, Viner RM. Effects of screentime on the health and well-being of children and adolescents: A systematic review of reviews. BMJ Open. 2019; 9: e023191.

16. Telama R. Tracking of physical activity from childhood to adulthood: A review. Obes Facts. 2009; 2: 187-195.

17. Telama R, Yang X, Viikari J, Välimäki I, Wanne O, Raitakari O. Physical activity from childhood to adulthood: A 21-year tracking study. Am J Prev Med. 2005; 28: 267-273.

18. Laible D, Thompson RA. Early socialization: A relationship perspective. In: Handbook of socialization: Theory and practice. New York: The Guilford Press; 2008.

19. Grusec JE. Socialization processes in the family: Social and emotional development. Annu Rev Psychol. 2011; 62: 243-269. 
20. Birch LL, Fisher JO, Grimm-Thomas K, Markey CN, Sawyer R, Johnson SL. Confirmatory factor analysis of the child feeding questionnaire: A measure of parental attitudes, beliefs and practices about child feeding and obesity proneness. Appetite. 2001; 36: 201-210.

21. Campbell K, Hesketh K, Davison KK, Crawford D, Jeffery RW, Ball K, et al. The role of parents in preventing child overweight and obesity: An ecological approach. In: Obesity epidemiology: From aetiology to public health. 2nd ed. Oxford: Oxford University Press; 2010. pp.299-320.

22. Scaglioni S, Salvioni M, Galimberti C. Influence of parental attitudes in the development of children eating behaviour. Br J Nutr. 2008; 99: S22-S25.

23. Faught E, Vander Ploeg K, Chu YL, Storey K, Veugelers PJ. The influence of parental encouragement and caring about healthy eating on children's diet quality and body weights. Public Health Nutr. 2016; 19: 822-829.

24. Davison KK, Cutting TM, Birch LL. Parents' activity-related parenting practices predict girls' physical activity. Med Sci Sports Exerc. 2003; 35: 1589-1595.

25. Eccles JS, Harold RD. Gender differences in sport involvement: Applying the Eccles' expectancyvalue model. J Appl Sport Psychol. 1991; 3: 7-35.

26. Eccles JS, Jacobs JE, Harold RD. Gender role stereotypes, expectancy effects, and parents' socialization of gender differences. J Soc Issues. 1990; 46: 183-201.

27. Trost SG, Sallis JF, Pate RR, Freedson PS, Taylor WC, Dowda M. Evaluating a model of parental influence on youth physical activity. Am J Prev Med. 2003; 25: 277-282.

28. Davison KK, Downs DS, Birch LL. Pathways linking perceived athletic competence and parental support at age 9 years to girls' physical activity at age 11 years. Res Q Exerc Sport. 2013; 77: 2331.

29. De Vet E, De Ridder DT, De Wit JB. Environmental correlates of physical activity and dietary behaviours among young people: A systematic review of reviews. Obes Rev. 2011; 12: e130e142.

30. Woodard E, Gridina N. Media in the home 2000: Fifth annual survey of parents and children [Internet]. Philadelphia: Annenberg Public Policy Center of the University of Pennsylvania; 2000. Available from:

https://cdn.annenbergpublicpolicycenter.org/Downloads/Media and Developing Child/medi asurvey/inhome.pdf.

31. He M, Piché L, Beynon C, Harris S. Screen-related sedentary behaviors: Children's and parents' attitudes, motivations, and practices. J Nutr Educ Behav. 2010; 42: 17-25.

32. Jago R, Davison KK, Thompson JL, Page AS, Brockman R, Fox KR. Parental sedentary restriction, maternal parenting style, and television viewing among 10-to 11-year-olds. Pediatrics. 2011; 128: e572-e578.

33. Davison K, Campbell K. Opportunities to prevent obesity in children within families: An ecological approach. In: Obesity prevention and public health. Oxford: Oxford University Press; 2005. pp.207-230.

34. Van Manen M. Researching Lived Experience: Human science for an action sensitive pedagogy. London: Althouse; 1990.

35. Bloomberg LD, Volpe M. Completing your qualitative dissertation: A road map from beginning to end. 2nd ed. Thousand Oaks: Sage Publications; 2012.

36. Glesne C. Becoming qualitative researchers. Boston: Pearson; 2006. 
37. Johnson R, Welk G, Saint-Maurice PF, Ihmels M. Parenting styles and home obesogenic environments. Int J Environ Res Public Health. 2012; 9: 1411-1426.

38. Lofland J, Lofland LH. Analyzing social settings: A guide to qualitative observation and analysis. Belmont: Wadsworth; 1995.

39. Wertz FJ. Phenomenological research methods for counseling psychology. J Couns Psychol. 2005; 52: 167-177.

40. Husserl E. Ideas: General introduction to pure phenomenology. New York: Collier Books; 1962.

41. Roth G, Assor A, Niemiec CP, Ryan RM, Deci EL. The emotional and academic consequences of parental conditional regard: Comparing conditional positive regard, conditional negative regard, and autonomy support as parenting practices. Dev Psychol. 2009; 45: 1119-1142.

42. Joussemet $M$, Landry R, Koestner R. A self-determination theory perspective on parenting. Can Psychol. 2008; 49: 194-200.

43. Ryan RM, Deci EL. Self-determination theory and the facilitation of intrinsic motivation, social development, and well-being. Am Psychol. 2000; 55: 68-78.

44. Nord CW. How involved are fathers in their children's schools [Internet]? Washington: US Department of Education, Office of Educational Research and Improvement, National Center for Education Statistics; 1998. Available from: http://nces.ed.gov/pubs98/98120.pdf.

45. Sax LJ, Gilmartin SK, Bryant AN. Assessing response rates and nonresponse bias in web and paper surveys. Res High Educ. 2003; 44: 409-432.

46. Ogden CL, Fakhouri TH, Carroll MD, Hales CM, Fryar CD, Li X, et al. Prevalence of obesity among adults, by household income and education-United States, 2011-2014. Morb Mortal Wkly Rep. 2017; 66: 1369-1373.

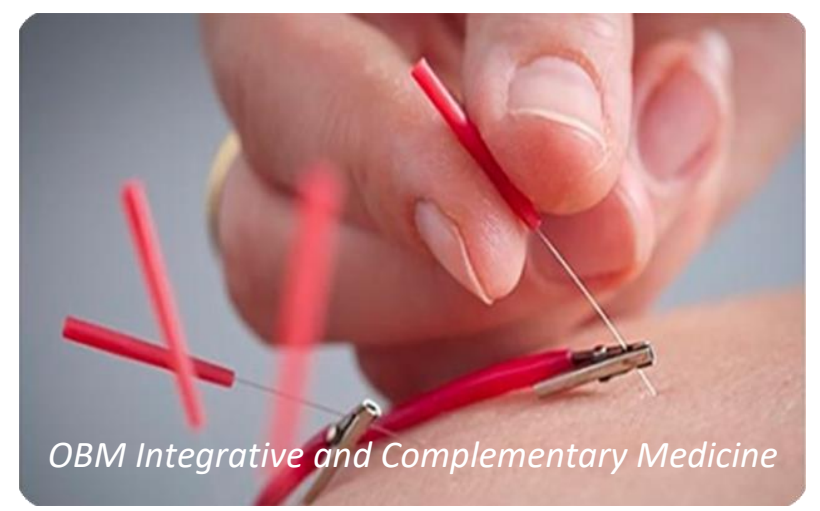

Enjoy OBM Integrative and Complementary Medicine by:

1. Submitting a manuscript

2. Joining in volunteer reviewer bank

3. Joining Editorial Board

4. Guest editing a special issue

For more details, please visit: http://www.lidsen.com/journals/icm 